\title{
Les caractères du système lipolytique de l'espèce Penicillium caseicolum.

\author{
Nature du système
}

\author{
par \\ G. LAMBERET et J. LENOIR \\ Laboratoire de Recherche de la Chaire de Technologie (I.N.R.A.) \\ Institut National Agronomique Paris-Grignon \\ 78850 Thiverval-Grignon
}

\section{INTRODUCTION}

L'étude de l'aptitude de Penicillium caseicolum à la lipolyse a permis de montrer qu'il existait, entre les souches, de grandes différences dans les niveaux de production de lipases exocellulaires et que, au sein de la population étudiée, deux sous-groupes pouvaient être distingués, les souches d'origine Neufchâtel formant un sousgroupe caractérisé par une plus grande aptitude à la production d'enzymes lipolytiques (Lamberet et Lenoir, 1972).

Les données sur les caractères de l'activité lipolytique de Penicillium caseicolum sont rares ; elles concernent plus particulièrement le niveau de production et le type d'action. Ainsi, Proks et Cingrosova (1962) ont noté une libération importante d'acides gras saturés à chaîne courte et une faible libération d'acides insaturés au cours de la croissance de cette espèce sur un milieu contenant de l'huile de beurre. En revanche, pour d'autres moisissures importantes en technologie fromagère, comme Penicillium roqueforti et Geotrichum candidum, les caractéristiques des lipases ont été précisées, notamment sur des préparations purifiées (Imamura et Kataoka, 1963, 1966 ; Eitenmiller, 1968 ; Marks et al., 1968 ; Tsujisaka et al., 1973). Par ailleurs, certains auteurs ont mis en évidence des variations dans la nature des acides gras libérés par les systèmes lipolytiques de différentes souches de ces deux espèces (Wilcox et al., 1955 ; Proks et al., 1956).

Le présent travail a été entrepris dans le but de définir les principales caractéristiques du système lipolytique de $P$. caseicolum et, éventuellement, vérifier s'il existe des différences dans les caractères de ce système en fonction de l'aptitude des souches à la production d'enzyme. 
Les essais ont été réalisés principalement sur des préparations enzymatiques exocellulaires, c'est-à-dire des filtrats de culture. Sur ces préparations ont été déterminées la stabilité du système ainsi que certaines de ses caractéristiques d'action, notamment les influences du $\mathrm{pH}$ et de la température sur la vitesse d'hydrolyse de la tributyrine et les aptitudes comparées à l'hydrolyse de la tributyrine et de la trioléine. Dans une série d'essais l'activité lipolytique endocellulaire a été déterminée à trois $\mathrm{pH}$ différents $(8,5-6,5$ et 4,5). Enfin, dans un essai de culture en milieu acide $(\mathrm{pH} 4$-5), la production d'enzyme endo ou exocellulaire, active à $\mathrm{pH}$ faible, a été recherchée.

\section{PROTOCOLE EXPERIMENTAL}

Le milieu et les conditions de culture, la préparation des extraits enzymatiques exocellulaires et les méthodes de détermination de l'activité lipolytique (méthode au pH-stat et méthode par mesure des variations de $\mathrm{pH}$ ) ont été décrits dans une note antérieure (Lamberet et Lenoir, 1972). Seules les modifications apportées sont mentionnées ci-après.

\section{Matériel d'étude et conditions de culture}

L'étude a porté sur huit souches d'origines diverses présentant des niveaux de production nettement différents (tab. 1).

TABLEAU 1. - Aptitude à la lipolyse des souches étudiées

\begin{tabular}{c|c|c|c|c|c|c|c|c}
\hline Souches & P 16 & PL 11 & P 12 & PA 15 & P 1 & PL 21 & PN 28 & PL 2 \\
\hline Activités lipolytiques* & 9 & 20 & 30 & 38 & 49 & 66 & 86 & 102 \\
\hline
\end{tabular}

* Activités lipolytiques déterminées par la méthode de mesure des variations de $\mathrm{pH}$. Résıltats exprimés en microéquivalents acide libérés par $\mathrm{mn}$ et par $\mathrm{ml}$ à $\mathrm{pH} 7,5$.

Les cultures en milieu alcalin ( $\mathrm{pH}$ initial 7,3-7,5) ont été réalisées soit en boîtes de Roux de $1 \mathrm{l}$ avec $250 \mathrm{ml}$ de milieu, soit en erlenmeyers de $150 \mathrm{ml}$, avec $40 \mathrm{ml}$ de milieu.

Pour les essais de culture à $\mathrm{pH}$ acide, le milieu, additionné d'un tampon succinate à la concentration $0,5 \mathrm{M}$, est réglé à $\mathrm{pH} 4,0$; il est réparti en erlenmeyers de $150 \mathrm{ml}$ à raison de $40 \mathrm{ml}$ par fiole et il est stérilisé dans les mêmes conditions que le milieu $\mathrm{pH}$ 7,5. Le temps 
de culture est de $12 \mathrm{j}$ et, au cours de l'incubation, le $\mathrm{pH}$ est maintenu, si besoin est, entre 4 et 5 par addition d'acide chlorhydrique concentré.

\section{Détermination de l'activité lipolytique}

\subsection{PréParations enzymatioues}

Les préparations enzymatiques exocellulaires sont constituées par les filtrats de culture ; elles peuvent être conservées pendant au moins $5 \mathrm{j}$ à $4^{\circ} \mathrm{C}$ sans qu'il y ait perte sensible d'activité.

Lors de la comparaison des activités sur tributyrine et sur trioléine, pour éviter la déstabilisation rapide de l'émulsion de trioléine due à la précipitation de phosphate de calcium dans le mélange de digestion, les phosphates ont été au préalable éliminés de la préparation enzymatique par addition à celle-ci d'un volume égal d'une solution de tampon tris $\mathrm{HCl} 0,05 \mathrm{M}$ à $\mathrm{pH} 9,4$ contenant du chlorure de calcium $(0,15 \mathrm{M})$. Le $\mathrm{pH}$ est ramené à 8,1 immédiatement après cette addition et le mélange est centrifugé pendant $5 \mathrm{mn}$ à $2500 \mathrm{~g}$ à $20^{\circ} \mathrm{C}$. Le surnageant constitue la nouvelle préparation enzymatique ; elle est diluée au 1/2 par rapport au filtrat de culture.

Les extraits enzymatiques endocellulaires sont constitués par la suspension du mycélium lavé, broyé dans une solution tampon 0,05 M - tampon tris $\mathrm{HCl}$ à $\mathrm{pH} 8,0$ pour les cultures en milieu alcalin, tampon succinate à $\mathrm{pH} 4,5$ pour les cultures en milieu acide - Le volume final de la suspension est porté à un volume égal à celui du milieu de culture.

Le broyage est réalisé à une température inférieure à $10^{\circ} \mathrm{C}$ par dispersion avec un appareil Ultra-Turrax, type TP $18 / 2 \mathrm{~N}$, à la vitesse maximale de rotation pendant $1 \mathrm{mn} 30$; cette opération est suivie d'un dégazage sous vide. La préparation enzymatique obtenue est conservée à $4^{\circ} \mathrm{C}$ jusqu'au moment des mesures.

La teneur en matière sèche de la préparation est déterminée par dessiccation de $20 \mathrm{ml}$ de la suspension à $102^{\circ} \mathrm{C}$ pendant $15 \mathrm{~h}$.

\subsection{Mesure de L'ACTIVITÉ}

Les activités lipolytiques ont été déterminées soit par mesure des variations de $\mathrm{pH}$, soit par mesure à $\mathrm{pH}$ constant (méthode au $\mathrm{pH}$-stat), selon les protocoles décrits antérieurement. La première méthode a été utilisée pour la détermination de l'activité résiduelle lors des essais de stabilité du système exocellulaire à $\mathrm{pH} 8,0$ à $37^{\circ} \mathrm{C}$ et pour l'étude de l'influence de la température sur l'activité du système. La seconde méthode a été mise en œuvre dans tous les autres essais avec les additifs ou modifications suivants :

\subsubsection{Préparation de l'émulsion de trioléine}

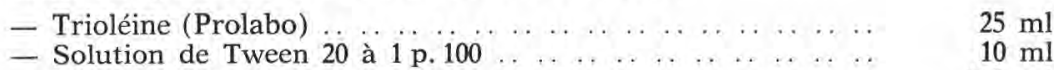

— Eau distillée q.s.p. .. . . . . . . . . . . . . . . . . . . . . $100 \mathrm{ml}$ 
L'émulsion est réalisée à l'Ultra-Turrax, avec agitation à la vitesse maximale pendant $2 \mathrm{mn}$. Elle est ensuite dégazée sous un vide faible.

\subsubsection{Composition du mélange de digestion}

- Emulsion de tributyrine ou de trioléine ... ..........

- Solution de tampon tris $\mathrm{HCl}, 0,01 \mathrm{M}, \mathrm{pH} \mathrm{8,2} \mathrm{contenant} \mathrm{du}$ chlorure de calcium $(0,025 \mathrm{M}) \ldots \ldots \ldots \ldots \ldots \ldots \ldots \ldots \ldots$

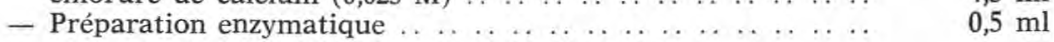

\subsubsection{Conditions des mesures}

Le $\mathrm{pH}$ de consigne choisi est maintenu automatiquement pendant 5 à $10 \mathrm{mn}$ par ajout d'une solution de soude $0,02 \mathrm{~N}$ ou $0,05 \mathrm{~N}$. Une unité-lipase correspond à la quantité d'enzymes qui libère, dans les conditions de l'essai, un micro-équivalent acide par minute.

Toutes les mesures d'activité ont été réalisées en double, sauf au cours du contrôle de la stabilité du système enzymatique à $\mathrm{pH}$ 5,5 où chaque résultat ne correspond qu'à une seule détermination.

\section{Essais de stabilité}

Dans les essais de stabilité en fonction du $\mathrm{pH}$ les préparations enzymatiques, maintenues à $4^{\circ} \mathrm{C}$, sont additionnées d'acide chlorhydrique ou de soude $0,2 \mathrm{~N}$ pour amener à $\mathrm{pH} 8,0$; elles sont ensuite ajustées à la valeur de $\mathrm{pH}$ voulue, dans l'intervalle 5,5-10,0, par addition de tampon tris-maléate $1 \mathrm{M}, \mathrm{pH} 2,9$ à 12 , dans la proportion de $1 \mathrm{ml}$ de tampon pour $5 \mathrm{ml}$ de filtrat de culture. La valeur de $\mathrm{pH}$ la plus élevée $(9,8)$ a été obtenue par une addition supplémentaire de soude $\mathrm{N}$.

Les préparations sont portées aux températures choisies, $30^{\circ} \mathrm{C}$ ou $37^{\circ} \mathrm{C}$, en tubes à essai pour les temps de chauffage de $30 \mathrm{mn}$, en tubes de verre de diamètre $3,5 \mathrm{~mm}$ pour les essais comportant des temps variables. L'action du chauffage est ensuite arrêtée en plongeant les tubes dans l'eau glacée. L'activité résiduelle est déterminée dans les 30 à $60 \mathrm{mn}$ qui suivent.

Dans les conditions des essais, les pertes d'activité des extraits conservés à $4^{\circ} \mathrm{C}$ aux différents $\mathrm{pH}$ sont négligeables.

\section{RESULTATS}

\section{Caractéristiques d'action}

\subsection{INFLUENCE du $\mathrm{pH}$}

L'influence du $\mathrm{pH}$ sur l'activité lipolytique a été éprouvée dans l'intervalle de $\mathrm{pH} 4,5-9,5$. Une correction a été apportée aux résultats obtenus aux $\mathrm{pH} 4,5,5,5$ et 6,5 afin de tenir compte de la dissociation incomplète de l'acide butyrique en milieu acide. Les pourcentages 
d'acide titré à ces valeurs de $\mathrm{pH}$ ont été calculés à partir de la constante d'ionisation $\left(1,484 \times 10^{-5}\right.$ à $\left.30^{\circ} \mathrm{C}\right)$. Les valeurs trouvées (33-82,5 et 98$)$ sont très voisines de celles déterminées expérimentalement par neutralisation de l'acide butyrique dans le mélange de digestion.

Les points de la figure 1 montrent les variations de l'activité lipolytique des huit souches en fonction du $\mathrm{pH}$ du mélange réactionnel. L'allure des différentes courbes est identique, aux erreurs expé-

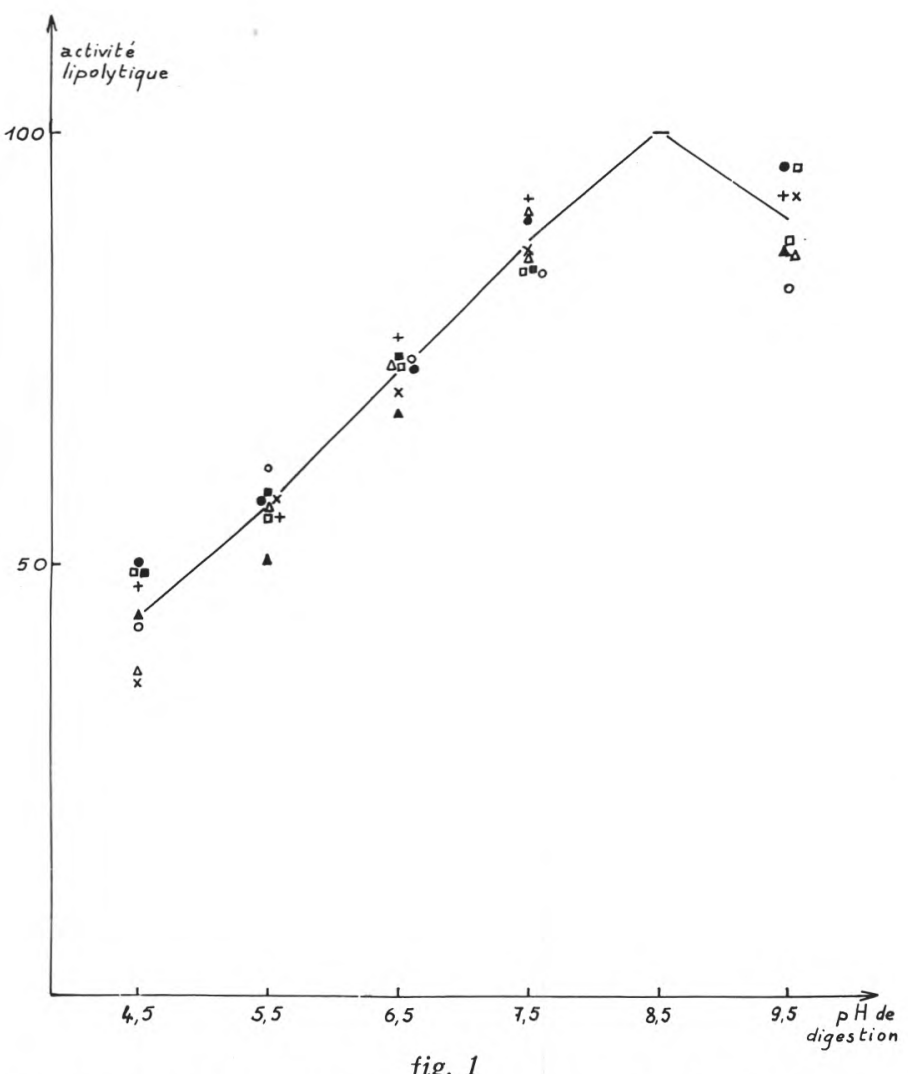

fig. 1

Influence du $\mathrm{pH}$ du mélange de digestion sur l'activité lipolytique de $P$. caseicolum

Activité mesurée à $30^{\circ} \mathrm{C}$ au pH-stat ; résultats exprimés en p. 100 de l'activité maximale.

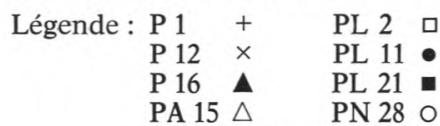

(courbe moyenne tracée en trait plein) 
rimentales près, et elle est régulière. L'activité maximale se situe à $\mathrm{pH} 8,5$ et les valeurs moyennes de l'activité aux $\mathrm{pH}$ 6,5 et 4,5 sont respectivement de 72 p. 100 et 45 p. 100 de l'optimum.

\subsection{INFLUENCE DE LA TEMPÉRATURE}

La température optimale d'action, déterminée par la méthode de mesure des variations de $\mathrm{pH}$, se situe aux environs de $30^{\circ} \mathrm{C}$. A $20^{\circ} \mathrm{C}$ l'activité est très proche de l'activité maximale, elle reste supérieure à 90 p. 100 de cette valeur. Au-dessus de $35^{\circ} \mathrm{C}$, l'inactivation est importante et à $50^{\circ} \mathrm{C}$ l'activité ne représente plus que 10 p. 100 de l'activité maximale (fig. 2).

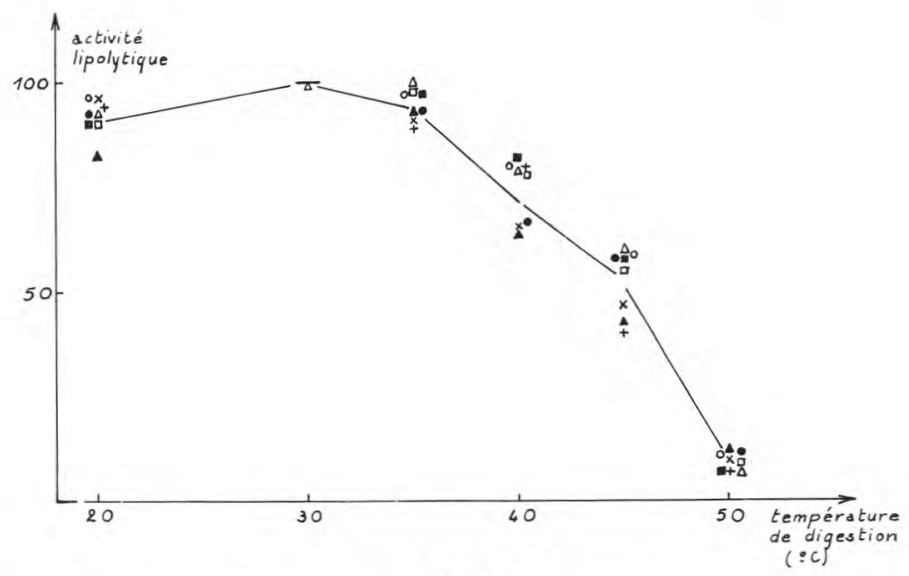

fig 2

Influence de la température du mélange de digestion sur l'activité lipolytique de $P$. caseicolum

Activité mesurée par la méthode de mesure des variations de $\mathrm{pH}$; résultats exprimés en p. 100 de l'activité maximale.

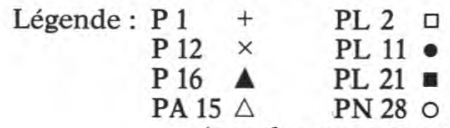

(courbe moyenne tracée en trait plein)

Les différences observées entre les souches sont faibles et non significatives.

\subsection{INFLUENCE DE LA NATURE DU SUBSTRAT}

Les activités ont été déterminées à $\mathrm{pH} 8,0$ par la méthode au $\mathrm{pH}$-stat sur les substrats tributyrine et trioléine. D'après Benzonana et Desnuelle (1968), l'acide oléique est totalement titré à cette valeur de $\mathrm{pH}$ en présence d'ions calcium en excès et de chlorure de sodium à la concentration $0,1 \mathrm{M}$. Dans le cas présent, cependant, la concen- 
tration en calcium est mal connue car la petite quantité d'acide oléique contenue dans la trioléine forme, avec le calcium, un sel insoluble qui doit être éliminé en portant le $\mathrm{pH}$ du mélange à 9-10. Le $\mathrm{pH}$ est ensuite ramené à 8,1 par addition d'acide chlorhydrique.

La mesure de l'activité est effectuée à $30^{\circ} \mathrm{C}$ par titrage avec de la soude $0,02 \mathrm{~N}$; la vitesse de la réaction est constante pendant les 5 premières minutes et elle est proportionnelle à la quantité d'extrait enzymatique.

Les rapports des vitesses d'hydrolyse des deux substrats, trioléine et tributyrine, ont, d'une souche à l'autre, des valeurs comparables (tab. 2). A pH 8, le système lipolytique de Penicillium caseicolum libère l'acide butyrique de la tributyrine cinq fois plus vite que l'acide oléique de la trioléine.

\section{TABLEAU 2}

Vitesses comparées de l'hydrolyse de la tributyrine et de la trioléine par le système lipolytique exocellulaire de $P$. caseicolum*

\begin{tabular}{c|c|c|c|c|c|c|c|c}
\hline Souches & P 1 & P 12 & P 16 & PA 15 & PL 2 & PL 11 & PL 21 & PN 28 \\
\hline $\begin{array}{c}\text { Vitesse relative } \\
\text { d'hydrolyse } \\
\text { de la trioléine }\end{array}$ & 18 & 18 & 25 & 19,5 & 19 & 19,5 & 18 & 19 \\
\hline
\end{tabular}

* Déterminations de l'activité réalisées au pH-stat, à $\mathrm{pH} 8,0$ et à $30^{\circ} \mathrm{C}$. La valeur 100 est attribuée à la vitesse d'hydrolyse de la tributyrine.

\section{Stabilité du système lipolytique}

\subsection{Stabilité EN FONCTion du pH}

La figure 3 a présente les résultats des déterminations de l'activité lipolytique à $\mathrm{pH} 8,5$ après maintien des préparations enzymatiques aux différents $\mathrm{pH}$ compris entre 5 et 10 pendant $30 \mathrm{mn}$ à $30^{\circ} \mathrm{C}$. Les courbes présentent une certaine concordance; la zone de stabilité maximale est située entre les $\mathrm{pH}$ de 7,3 et 8,5 pour les huit préparations et l'on constate que, de part et d'autre de cette zone, aux pH 6,5 et 9, les pertes d'activité sont faibles, en moyenne, respectivement, 20 et 8 p. 100. A pH 5,8, toutes les souches présentent le même taux d'inactivation, proche de 80 p. 100. A pH 9,8, la dispersion des résultats est assez grande ; les souches P 16, PN 28 et surtout PL 2, ont un taux d'inactivation nettement supérieur à celui des autres souches.

Les courbes de la figure $3 \mathrm{~b}$ représentent les moyennes des activités résiduelles mesurées à $\mathrm{pH} 8,5$ et 5,5 . On remarque que les valeurs extrêmes, aux pH 5,8 et 9,8 , sont pratiquement identiques ; 


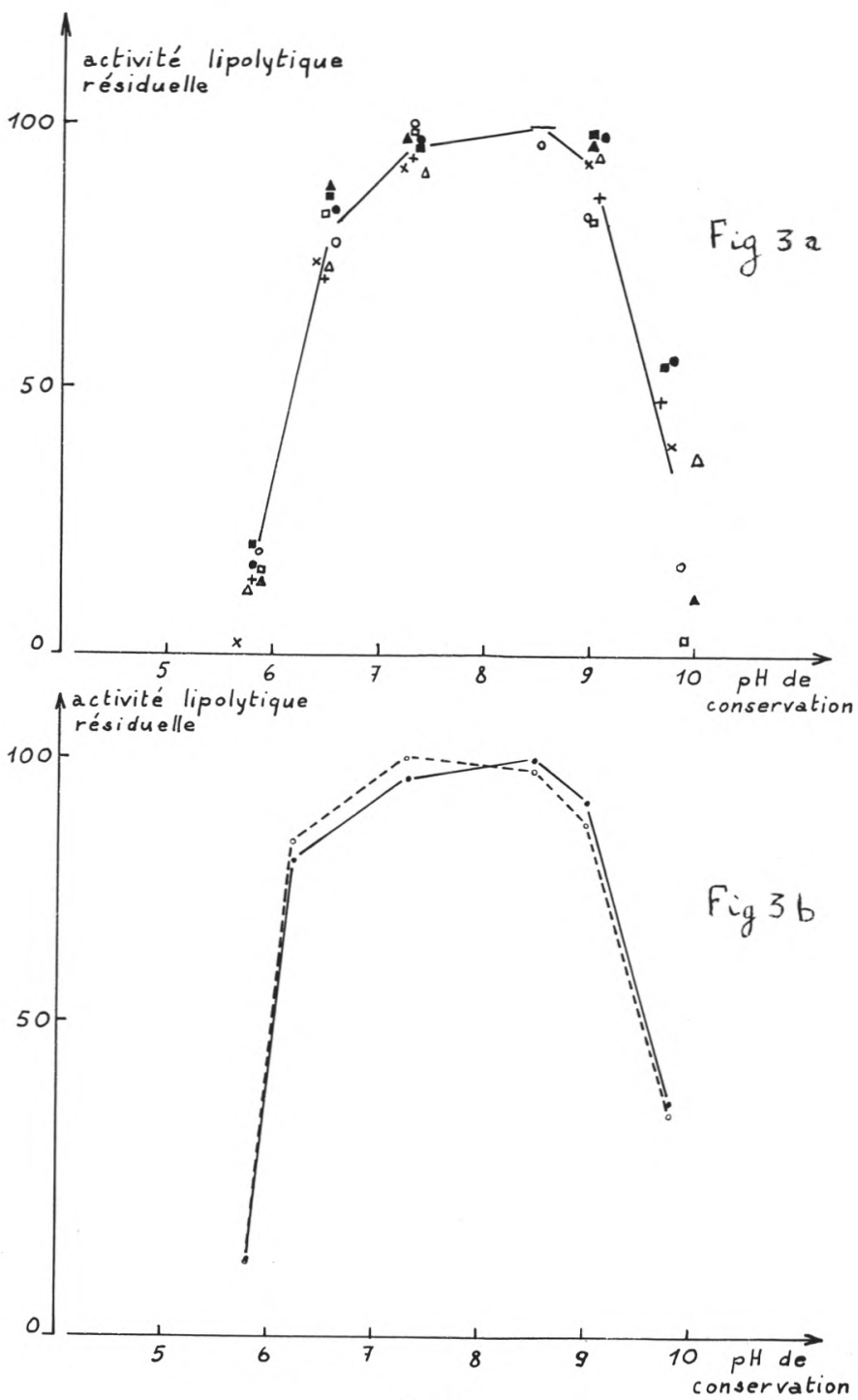

fig. 3

Stabilité en fonction du $\mathrm{pH}$

Préparation enzymatiłue en tampon tris-maléate $M / 6$, maintenue $30 \mathrm{mn}$ à $30^{\circ} \mathrm{C}$ aux différents $\mathrm{pH}$; activités mesurées au pH-stat ; résultats exprimés en p. 100 de l'activité maximale.
Légende : $\mathrm{P} 1+$
PL 2
$\mathrm{P} 12 \times$
PL $11 \bullet$
P $16 \triangle$
PL 21 -
PA $15 \triangle$ PN 280

Figure $3 \mathrm{a}$ : activités mesurées à $\mathrm{pH}$ 8,5. Courbe moyenne tracée en trait plein.

Figure $3 \mathrm{~b}$ : courbes moyennes des activités mesurées à $\mathrm{pH} 8,5$ (trait plein) et à pH 5,5 (en tirets). 

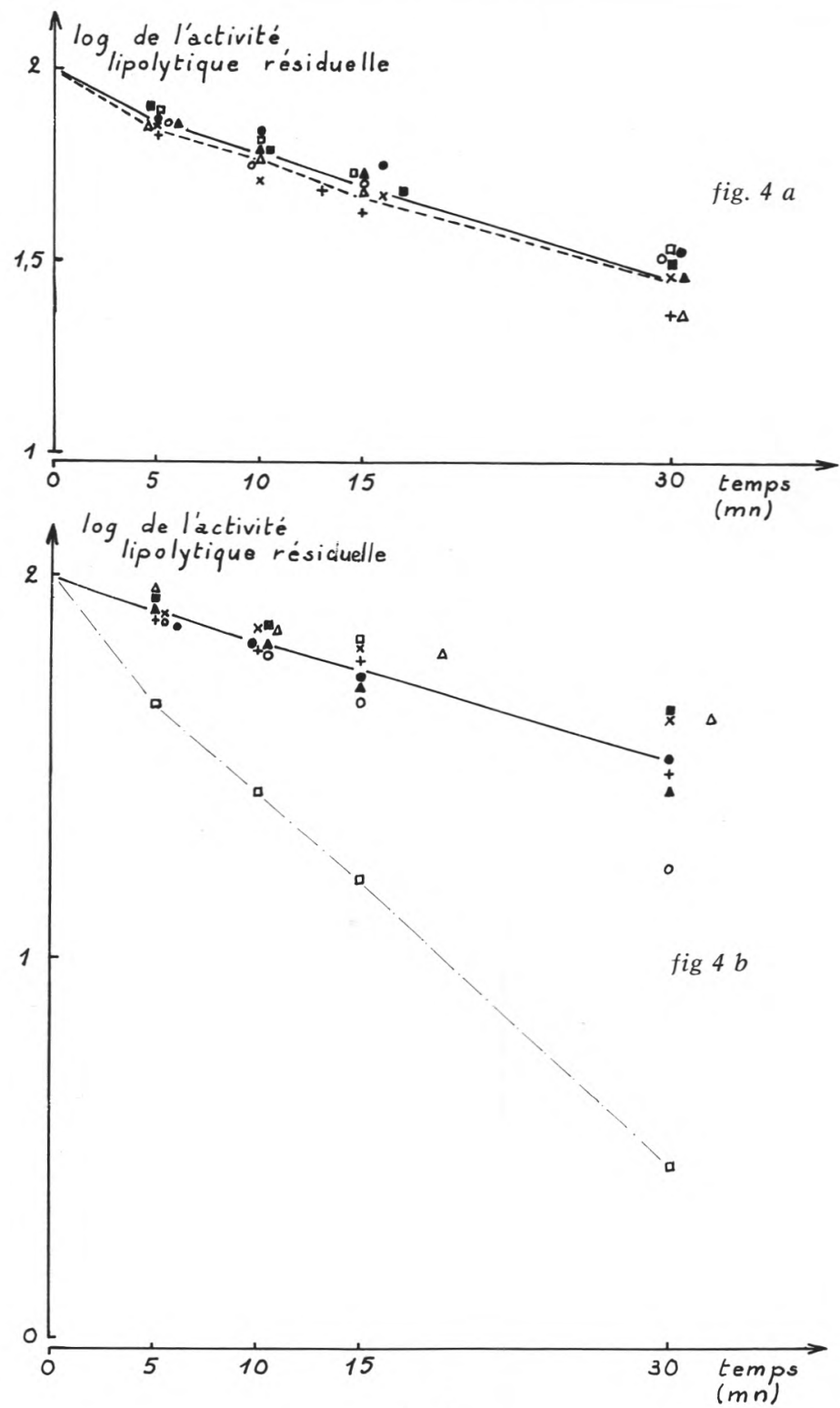

fig. 4

Stabilité en fonction du temps

Figure 4 a (en haut) : pH 6,05.

Figure $4 \mathrm{~b}$ (en bas) : pH 9,8.

Préparations enzymatiques en tampon tris-maléate $\mathrm{M} / 6$, portées à $30^{\circ} \mathrm{C}$ pendant un temps variable $(5$ à $30 \mathrm{mn}$ ).

Activités résiduelles déterminées au pH-stat. Résultats exprimés en p. 100 de l'activité initiale.

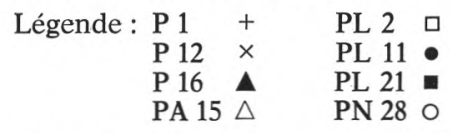

En trait plein : courbes moyennes des activités mesurées à $\mathrm{pH} 8,5$.

En tirets :

figure $4 \mathrm{a}$ : courbe moyenne des activités mesurées à $\mathrm{pH} 5,5$

figure $4 \mathrm{~b}$ : courbe relative à la souche PL 2 . 
de légères différences sont observées pour les autres valeurs du pH. L'activité résiduelle mesurée à $\mathrm{pH} 5,5$ est légèrement plus grande que celle mesurée à $\mathrm{pH} 8,5$ dans la zone des $\mathrm{pH}$ acides et son maximum est atteint à $\mathrm{pH} 7,3$; elle est plus faible dans la zone des $\mathrm{pH}$ alcalins.

Les courbes de la figure 4, tracées en coordonnées semi-logarithmiques, représentent l'allure de l'inactivation en fonction du temps à $\mathrm{pH} \mathrm{6,05} \mathrm{(fig.} 4 \mathrm{a}$ ) et à $\mathrm{pH} 9,8$ (fig. 4 b). Après maintien à $\mathrm{pH} \mathrm{6,05,}$ les activités résiduelles sont peu différentes les unes des autres; la dispersion des points est proche de celle qui peut être relevée sur la figure 3 a dans la zone des $\mathrm{pH}$ acides. Les courbes moyennes correspondant aux activités mesurées aux $\mathrm{pH}$ de 8,5 et 5,5 ont des allures comparables et si la stabilité mesurée à $\mathrm{pH} 5,5$ apparaît très légèrement plus faible, cette différence n'est guère significative.

A pH 9,8, les courbes de déstabilisation ont la même allure mais on observe une plus grande dispersion des résultats, la préparation enzymatique de la souche PL 2 paraissant être beaucoup plus sensible.

\subsection{Stabilité a $37^{\circ} \mathrm{C}$ en Fonction du temps}

L'inactivation thermique du système lipolytique a été déterminée à la température de $37^{\circ} \mathrm{C}$ dans la zone de $\mathrm{pH}$ correspondant à la stabilité maximale ( $\mathrm{pH} \mathrm{8,0).} \mathrm{A} \mathrm{l'exception} \mathrm{de} \mathrm{la} \mathrm{souche} \mathrm{P} 16$, qui se distingue assez nettement, les différences entre les souches sont assez faibles (fig. 5) et l'on constate un degré d'inactivation élevé, supérieur à $93 \mathrm{p} .100$, après $15 \mathrm{mn}$.

\section{Activité lipolytique endocellulaire}

Les résultats des déterminations de l'activité lipolytique sur les mycéliums broyés, aux $\mathrm{pH}$ de 4,5-6,5 et 8,5, sont groupés dans le tableau 3. Les valeurs de l'activité à $\mathrm{pH} 8,5$, rapportée au $\mathrm{mg}$ de mycélium sec, peuvent être d'une souche à l'autre très différentes. Ainsi, les niveaux de production, en unités lipase par $\mathrm{mg}$, oscillent de 0,72 (souche P 16) à 12,93 (souche PN 28). Observons cependant que la reproductibilité des résultats est imparfaite, des variations atteignant une amplitude de 1 à 2 ont pu être constatées sur des cultures différentes d'une même souche ; il apparaît donc que les paramètres contrôlant la production d'enzymes ou leur extraction sont insuffisamment maîtrisés.

Les rapports des niveaux de production des enzymes endocellulaires et des enzymes exocellulaires varient suivant les souches de 0,13 à 0,7 .

L'influence du $\mathrm{pH}$ sur l'activité endocellulaire est proche de celle observée pour l'activité exocellulaire. Comparées à l'activité à $\mathrm{pH} 8,5$ les activités endocellulaires aux $\mathrm{pH}$ 6,5 et 4,5 varient peu d'une souche à l'autre; les valeurs obtenues sont respectivement proches de 70 et 48 ; ce sont des valeurs voisines de celles notées pour les activités exocellulaires ( 72 et 43 ). 


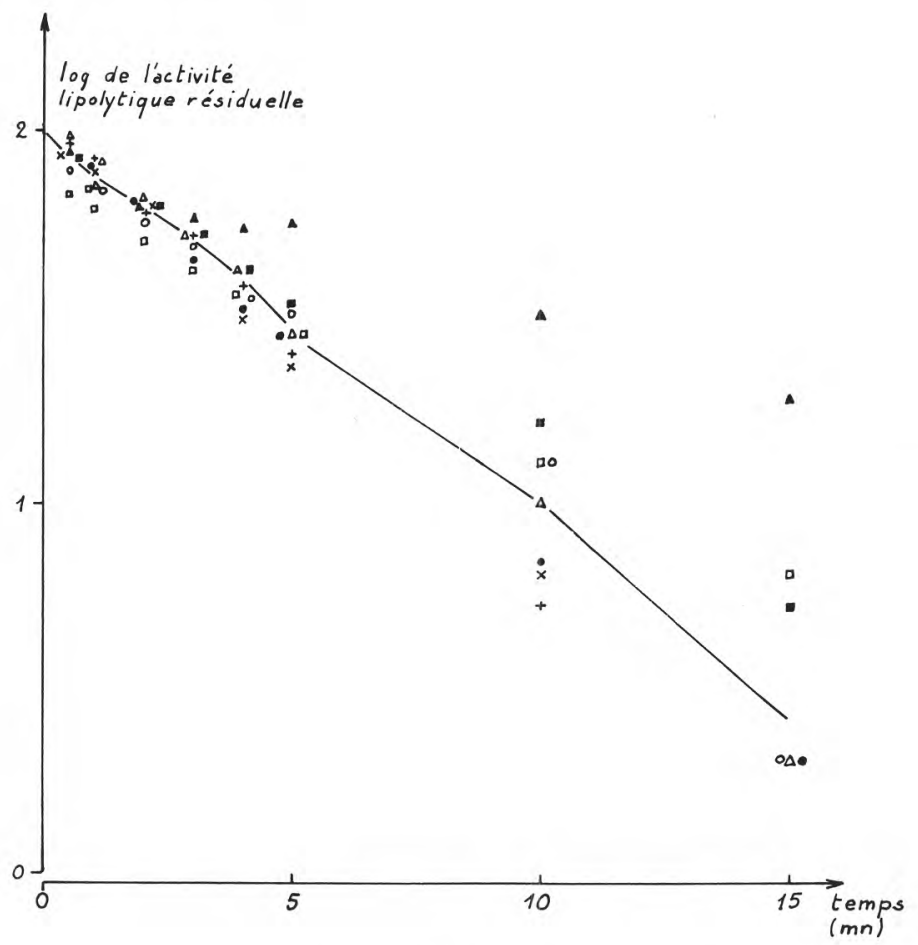

fig. 5

Stabilité à $37^{\circ} \mathrm{C}$ et $\mathrm{pH} 8,0$ en fonction du temps

Activités résiduelles déterminées par la méthode de mesure des variations de $\mathrm{pH}$; résultats exprimés en p. 100 de l'activité initiale.

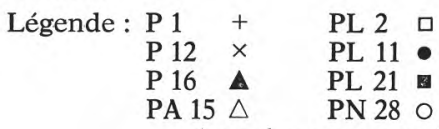

(courbe moyenne tracée en trait plein)

\section{Production de lipase en milieu acide}

L'essai a été réalisé sur six cultures (souches P 16, P 1, PA 15, PL 2, PL 21 et PN 28). Dans les conditions opératoires retenues, aucune activité lipolytique exocellulaire n'a été décelée, aussi bien dans les déterminations à $\mathrm{pH} 4,5$ que celles à $\mathrm{pH} 8,5$. Avec certaines souches (PL 2, PL 21, PN 28), une activité endocellulaire appréciable a été mise en évidence (tab. 4) mais les rapports activité à $\mathrm{pH} \mathrm{8,5/}$ activité à $\mathrm{pH} 4,5$ sont très proches de ceux qui caractérisent le système lipasique produit en milieu alcalin. 
$T A B L E A U 3$ : Activités lipolytiques endocellulaires

\begin{tabular}{l|c|c|c} 
& & \multicolumn{2}{|c}{ Activités relatives ${ }^{* *}$} \\
Souches & $\begin{array}{c}\text { Activités lipolytiques* } \\
\text { à pH 8,5 }\end{array}$ & à pH 6,5 & à pH 4,5 \\
\cline { 2 - 3 } & & & \\
P 1 & 1,9 & 70,0 & 48,0 \\
P 12 & 2,2 & 70,4 & 44,8 \\
P 16 & 0,7 & 60,5 & 55,9 \\
PA 15 & 1,7 & 71,7 & 52,0 \\
PL 2 & 3,0 & 67,2 & 49,2 \\
PL 11 & 0,7 & 67,2 & 49,6 \\
PL 21 & 4,9 & 75,8 & 44,6 \\
PN 28 & 12,9 & 75,3 & 36,7 \\
\hline
\end{tabular}

Conditions de culture : milieu Czapek-trypticase à $\mathrm{pH}$ 7,5 réparti en erlenmeyers de $150 \mathrm{ml}$, incubation $12 \mathrm{j}$ à $20^{\circ} \mathrm{C}$ sans agitation.

* Activités lipolytiques mesurées au pH-stat ; résultats exprimés en unitéslipase par mg de mycélium sec.

** Valeur 100 correspondant aux activités à pH 8,5.

TABLEAU 4 : Production de lipase en milieu acide

\begin{tabular}{l|c|c}
\hline Souches & $\begin{array}{c}\text { Activité lipolytique* } \\
\text { à pH 8,5 }\end{array}$ & $\begin{array}{c}\text { Activité lipolytique relative } \\
\text { à pH } 4,5^{* *}\end{array}$ \\
\hline & & 45 \\
PL 2 & 0,4 & 38 \\
PL 21 & 0,5 & 48 \\
PN 28 & 1,6 & \\
\hline
\end{tabular}

Conditions de culture : milieu Czapek-trypticase tamponné à $\mathrm{pH}$ 4-5, incubation $12 \mathrm{j}$ à $20^{\circ} \mathrm{C}$ sans agitation.

* Activités lipolytiques mesurées au pH-stat ; résultats exprimés en unités lipase par mg de mycélium sec.

** Valeur 100 correspondant à l'activité lipolytique à pH 8,5. 


\section{DISCUSSION}

Les niveaux de production de lipases des souches de Penicillium caseicolum étudiées sont très différents, leurs systèmes lipolytiques exocellulaires n'en présentent pas moins des caractéristiques qui sont dans l'ensemble très voisines, tant du point de vue de la stabilité que des conditions optimales d'action.

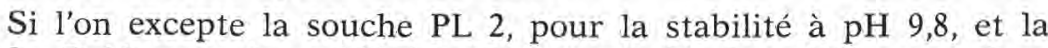
souche $\mathrm{P} 16$, pour la stabilité à $37^{\circ} \mathrm{C}$ à $\mathrm{pH} 8,0$, les différences dans les caractères de l'activité enzymatique des diverses souches peuvent en effet être considérées comme négligeables. On peut d'ailleurs observer que la souche $\mathrm{P} 16$ ne produit qu'une faible quantité d'enzymes et dans ces conditions les activités résiduelles exprimées en p. 100 ne peuvent qu'être approximatives ; quant à la souche PL 2, tous les autres caractères de son activité s'identifient bien à ceux des diverses souches essayées. Il semble donc permis d'admettre que toutes les souches de $P$. caseicolum produisent le même système lipolytique.

Les courbes d'activité en fonction du $\mathrm{pH}$ montrent une diminution de l'activité au-dessus de $\mathrm{pH} 8,5$. Elle peut s'expliquer par la dénaturation du système lipolytique qui est déjà marquée à $\mathrm{pH} \quad 9,5$ et qui s'amplifie aux pH plus élevés (fig. 3 a) ; aucune détermination d'activité n'a pu cependant être faite à $\mathrm{pH}$ supérieur à 9,5 en raison de la déstabilisation de l'émulsion, déstabilisation due probablement à certains composants du milieu de culture, car la même difficulté n'a pas été rencontrée avec les préparations enzymatiques purifiées.

Dans la zone des $\mathrm{pH}$ acides, 4,5-5,5, le système conserve une activité encore appréciable bien que sa stabilité soit faible aux pH inférieurs à 6. Par ailleurs, l'allure des courbes enregistrées au pH-stat montrent que, même à $\mathrm{pH} 4,5$, le taux de lipolyse reste pratiquement constant pendant les 5 à $10 \mathrm{mn}$ que dure la mesure d'activité. Il est donc probable que la stabilité du système lipolytique de $P$. caseicolum se trouve sensiblement accrue en présence du substrat.

Dans la détermination de l'influence de la température sur la vitesse d'hydrolyse de la tributyrine, on peut considérer que l'activité lipolytique, estimée par la méthode de mesure des variations de $\mathrm{pH}$, correspond à la vitesse initiale de la réaction, au moins jusqu'à $30^{\circ} \mathrm{C}$, car le temps d'incubation est relativement court (5 à $7 \mathrm{mn}$ ). La température optimale d'action apparaît donc assez faible. Elle peut être comparée à celles observées sur un certain nombre de lipases fongiques : $25^{\circ} \mathrm{C}$ pour Aspergillus niger, (Fukumoto et al., 1963), $37^{\circ} \mathrm{C}$ pour $P$. roqueforti (Eitenmiller, 1968 ), $40^{\circ} \mathrm{C}$ pour Geotrichum candidum (Tsujisaka et al., 1973).

De plus avec le système lipolytique de Penicillium caseicolum, le facteur température paraît n'avoir, dans l'intervalle $20^{\circ} \mathrm{C}-30^{\circ} \mathrm{C}$, qu'une 
faible influence sur la vitesse de la lipolyse puisque celle-ci est encore à $20^{\circ} \mathrm{C}$ supérieure à $90 \mathrm{p} .100$ de la valeur maximale. On peut donc penser que, même aux températures modérées, l'inactivation de l'enzyme est déjà importante et qu'elle neutralise l'effet de l'augmentation de température sur la vitesse propre de la réaction.

Les résultats obtenus par action de la lipase sur des substrats purs confirment la libération préférentielle des acides gras courts qui avait été déjà rapportée par Proks et Cingrosova (1962). On observe en effet que la tributyrine est hydrolysée beaucoup plus rapidement que la trioléine; le rapport des deux vitesses de lipolyse, proche de 5 , est très voisin de celui obtenu par Eitenmiller (1968) avec la lipase partiellement purifiée de Penicillium roqueforti.

Sans doute est-il difficile de comparer des résultats obtenus dans des conditions différentes, les uns avec une lipase purifiée sur un substrat complexe, l'huile de beurre, les autres avec des préparations brutes sur tributyrine; on peut cependant remarquer une certaine parenté entre les caractères des systèmes lipasiques de $P$. roqueforti et de $P$. caseicolum. La lipase de $P$. roqueforti a également son $\mathrm{pH}$ optimal en zone faiblement alcaline et une action préférentielle sur les triglycérides contenant des acides gras courts ; sa température optimale est $37^{\circ} \mathrm{C}$, c'est-à-dire sensiblement supérieure à celle du système de $P$. caseicolum et son activité est très diminuée par un abaissement de la température à $25^{\circ} \mathrm{C}$ ou $30^{\circ} \mathrm{C}$; sa stabilité thermique est aussi plus grande, après $10 \mathrm{mn}$ à $37^{\circ} \mathrm{C}$ la perte d'activité ne dépasse guère 30 p. 100 . Ces différences de sensibilité à l'action de la température ne sont pas nécessairement dues à des différences dans la nature des systèmes enzymatiques, elles pourraient être liées à des différences dans les degrés de pureté des préparations.

L'étude du système lipolytique endocellulaire de $P$. caseicolum n'a pas été suffisamment approfondie pour que des conclusions définitives puissent être tirées. On peut noter que cette activité est assez importante et elle peut être proche de l'activité exocellulaire ; elle varie aussi beaucoup d'une souche à l'autre (amplitude de 1 à 18) et, globalement, dans le même sens que cette dernière. Par ailleurs, l'influence du $\mathrm{pH}$, dans l'intervalle 4,5-8,5, sur les deux activités est comparable. Ces observations devront cependant être confirmées car les conditions de production des systèmes exo et endocellulaires peuvent être différentes. Ainsi Imamura et Kataoka (1963) rapportent, pour les lipases de $P$. roqueforti, une production maximale à $25^{\circ} \mathrm{C}$ pour l'enzyme exocellulaire, entre $7^{\circ} \mathrm{C}$ et $20^{\circ} \mathrm{C}$ pour l'enzyme endocellulaire ; mais ces deux lipases se distinguent également par leur $\mathrm{pH}$ optimal d'action sur la tributyrine (respectivement 7,5 et 6,5) et par leur spécificité.

Les essais réalisés sur plusieurs souches n'ont pas permis de mettre en évidence chez $P$. caseicolum l'existence d'une lipase présen- 
tant un $\mathrm{pH}$ optimal d'action en milieu acide. Observons à ce sujet que chez deux espèces voisines, $P$. camemberti et $P$. nalgiovensis, Dolezalek et Minarik (1968) ont montré qu'aucune activité lipolytique ne se manifestait à $\mathrm{pH}$ inférieur à 5,5 à $20^{\circ} \mathrm{C}$ dans les 10 premiers jours d'une culture sur milieu contenant de la matière grasse de lait mais qu'une telle activité pouvait apparaître tardivement, après 20 ou $30 \mathrm{j}$.

On peut finalement conclure que les différentes souches de $P$. caseicolum, quelle que soit leur aptitude à la lipolyse, synthétisent un même système lipolytique de $\mathrm{pH}$ optimal 8,5 qui, d'après les caractères établis sur des préparations non purifiées, se rapprocherait de celui de $P$. roqueforti.

\section{Rés u m é}

Les caractères du système lipolytique de Penicillium caseicolum ont été déterminés sur des préparations enzymatiques brutes obtenues à partir de cultures de huit souches présentant des aptitudes à la lipolyse nettement différentes.

Les systèmes lipolytiques produits par ces souches présentent des caractères similaires. Leur $\mathrm{pH}$ optimal d'action est 8,5 à $30^{\circ} \mathrm{C}$ et à $\mathrm{pH} 4,5$ l'activité est voisine de 45 p. 100 de l'activité maximale. La température optimale d'action est de $30^{\circ} \mathrm{C}$ mais à $20^{\circ} \mathrm{C}$ l'enzyme reste encore très active. L'hydrolyse de la tributyrine s'effectue à une vitesse cinq fois plus grande que celle de la trioléine.

A $30^{\circ} \mathrm{C}$, les préparations sont stables dans l'intervalle de $\mathrm{pH} 7-8,5$; après maintien $30 \mathrm{mn}$ à $\mathrm{pH} 6$ l'activité résiduelle est proche de 30 p. 100 de l'activité initiale. La stabilité thermique est faible : à $\mathrm{pH} 8,0$, après conservation $15 \mathrm{mn}$ à $37^{\circ} \mathrm{C}$, le degré d'inactivation est supérieur à 90 p. 100 .

Les activités lipolytiques endocellulaires sont proches des activités exocellulaires par leur niveau et leur $\mathrm{pH}$ d'action. Aucune lipase présentant un $\mathrm{pH}$ optimal d'action en milieu acide n'a été mise en évidence sur les préparations endo et exocellulaires.

Il apparaît finalement que les souches qui se distinguent par leur niveau de production produisent le même système lipolytique.

\section{S u m m a r y}

The characteristics of the lipolytic system of Penicillium caseicolum have been tested with crude enzyme preparation obtained from cultures of 8 strains showing different abilities to produce extracellular lipases.

The systems produced by these strains reveal similar characteristics ; the optimal $\mathrm{pH}$ is 8.5 at $30^{\circ} \mathrm{C}$ and at $\mathrm{pH} 4.5$, nearly $45 \mathrm{p} .100$ of the maximal activity is still measured. Tributyrin hydrolysis occurs at 5 times higher rate than that of triolein. 
At $30^{\circ} \mathrm{C}$ enzyme preparations are stable within a $\mathrm{pH}$ range of 7-8.5 and during $30 \mathrm{mn}$ incubation at $\mathrm{pH} 6.0,70 \mathrm{p} .100$ of the initial activity is lost. The heat stability is low : at $37^{\circ} \mathrm{C}$ and $\mathrm{pH} 8.0$ less than $15 \mathrm{mn}$ are needed to reduce the activity to $10 \mathrm{p} .100$ of the original.

Endocellular lipolytic activities are close to exocellular ones with regard of their amount of production and their $\mathrm{pH}$ of action. No lipase having an acid optimal $\mathrm{pH}$ from either endo or exocellular preparation has been found.

It is conclued that strains which differ by their level of production produce the same lipolytic system.

Reçu pour publication en août 1975.

\section{Références bibliographiques}

Benzonana (G.) and Desnuelle (P.) (1968). - Action of some effectors on the hydrolysis of long-chain triglycerides by pancreatic lipase. Biochim. Biophys. Acta, 164, 47-58.

Dolezalek (J.) et Minarik (R.) (1968). - Influence de l'acidité du milieu et de la présure sur l'activité enzymatique de Penicillium camemberti et de Penicillium nalgiovensis. Sb. vys. Sk. Chem. - Technol. Praze, E 20, 67-77.

EITENMILleR (R. R.) (1968). - Production and properties of a Penicillium roqueforti lipase. Thèse pour le "Degree of Master of Science " Department of Food Science and Technology, University of Nebraska, Lincoln.

Fukumoto (J.), Iwai (M.) and TsujisaKa (Y.) (1963). - Studies on lipase. I. Purification and crystallization of a lipase secreted by Aspergillus niger. J. Gen. Appl. Microbiol., 9 (3), 353-361.

ImAMURA (T.) and KatAoKA (K.) (1963). - Biochemical studies on the manufacturing of Roquefort - type cheese. II. Characteristics of lipases produced by Penicillium roqueforti. Jap. J. Zootech. Sc., 34 (5), 349-353.

IMAMURA (T.) and KataоKA (K.) (1966). - Biochemical studies on the manufacturing of Roquefort - type cheese. III. Isolation of lipases from mould-culture. Jap. J.. Dairy Sci., 15 (6), A 138-142.

Lamberet (G.) et Lenorr (J.) (1972). - Aptitude de l'espèce Penicillium caseicolum à la production d'enzymes lipolytiques. Le Lait, 52 (513-514), 175-192.

Marks (T. A.), Quinn (J. G.), SAmpugna (J.) and Jensen (R. G.) (1968). - Studies on the specificity of a lipase system from Geotrichum candidum. Lipids, 3 (2), 143-146.

Proks (J.), DolezaleK (J). and Pech (Z.) (1956). - Biochemical studies about Penicillium roqueforti. C.R. XIVe Cong. Int. Lait., vol, II, 401-409.

Proks (J.) and Cingrosova (K.) (1962). - The influence of Penicillium camemberti and Penicillium caseicolum on proteolytic and lipolytic changes in the ripe-

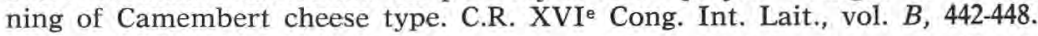

TSUJisaKa (Y.), IWAI (M.) and Tominaga (Y.) (1973). - Purification, crystallization and some properties of lipase from Geotrichum candidum Link. Agr. Biol. Chem., 37 (6), 1457-1464.

Wilcox (J. C.), NeLSON (W. O.) and Wood (W. A.) (1955), - The selective release of volatile acids from butterfat by microbial lipases. J. Dairy Sci., 38, 775-781. 